\title{
Visual loss and foveal lesions in Usher's syndrome
}

\author{
GERALD FISHMAN, VICTORIA VASQUEZ, MARLENE FISHMAN, AND DALE \\ BERGER $^{1}$
}

From the Department of Ophthalmology, University of Illinois Eye and Ear Infirmary, Chicago, and the ${ }^{1}$ Chicago Lighthouse for the Blind

SUMMARY Forty-eight patients with Usher's syndrome were assessed for the degree of visual loss, extent of lens opacities, and presence of foveal lesions. Twenty-one patients $(44 \%)$ showed either atrophic or cystic-appearing bilateral foveal changes. Despite having foveal lesions and variable degrees of lens opacities most patients maintained central visual acuity of $20 / 60$ or better until their middle $30 \mathrm{~s}$.

von Graefe (1858) was first to report the association of retinitis pigmentosa and congenital hearing loss. Subsequent reports, including those by Usher (1914) and Hallgren (1959), emphasised additional though variable findings that include cataracts, speech disorder, mental retardation, psychosis, and vestibular ataxia.

Although isolated reports refer to loss of central vision with increasing age in patients with Usher's syndrome (Merin et al., 1974; Bergsma, 1976; Abraham et al., 1977), actuarial data documenting progressive visual loss and quantitative analyses of contributing factors (for example, foveal lesions and cataracts) are not, to our knowledge, available in the ophthalmic literature.

This study analyses loss of central vision in patients with Usher's syndrome and correlates changes within the lens and fovea with the degree of central visual loss.

\section{Patients and methods}

Forty-eight consecutive patients with Usher's syndrome were prospectively examined and included in this study. All patients complained to some degree of nyctalopia and all showed abnormal rod function by electroretinography. The majority had bone-spicule-like pigmentary changes within the retina. Although it varied in severity, all the patients had some congenital deficiency of neurosensory hearing.

Each patient underwent a careful fundus examina-

Address for reprints: Dr G. A. Fishman, University of Illinois Eye and Ear Infirmary, $1855 \mathrm{~W}$. Taylor Street, Chicago, Ilinois 60612, USA

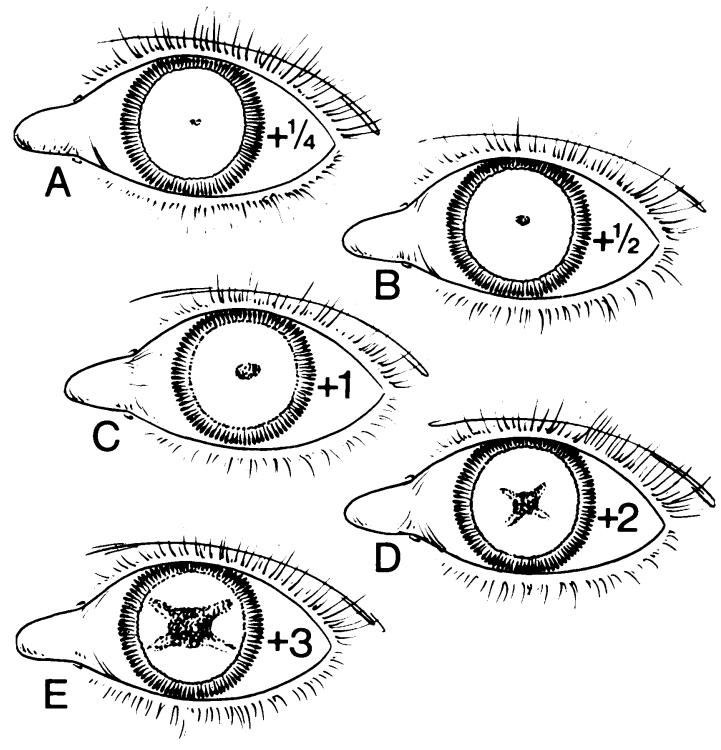

Fig. 1 Degrees of increasing posterior subcapsular lens opacities $(A-E)$

tion with emphasis on slit-lamp contact lens and direct and indirect ophthalmoscopy. Lens changes were assessed by slit-lamp biomicroscopy and categorised as to the presence and degree of posterior subcapsular lens opacities by criteria shown in Fig. 1 . Qualitative categorisation of foveal changes was accomplished by using previously published descriptions of the types of foveal lesions seen in patients with retinitis pigmentosa (Fishman et al., 1977a, b). Fluorescein angiograms were obtained by standard procedures following the rapid injection of $5 \mathrm{ml}$ of 
$10 \%$ sodium fluorescein into an antecubital vein. In addition all patients had colour fundus photographs and a cycloplegic refraction to obtain the best corrected visual acuity.

\section{Results}

Table 1 shows the age, sex, vision, extent of lens changes, and type of foveal lesion seen in the 48 patients. The fovea is defined here as a $1.5-\mathrm{mm}$ area within the macula that projects approximately $6^{\circ}$ on central visual field testing with the foveola at its centre. We define the macula as an area of approxi-

Table 1 Patient data and clinical findings*

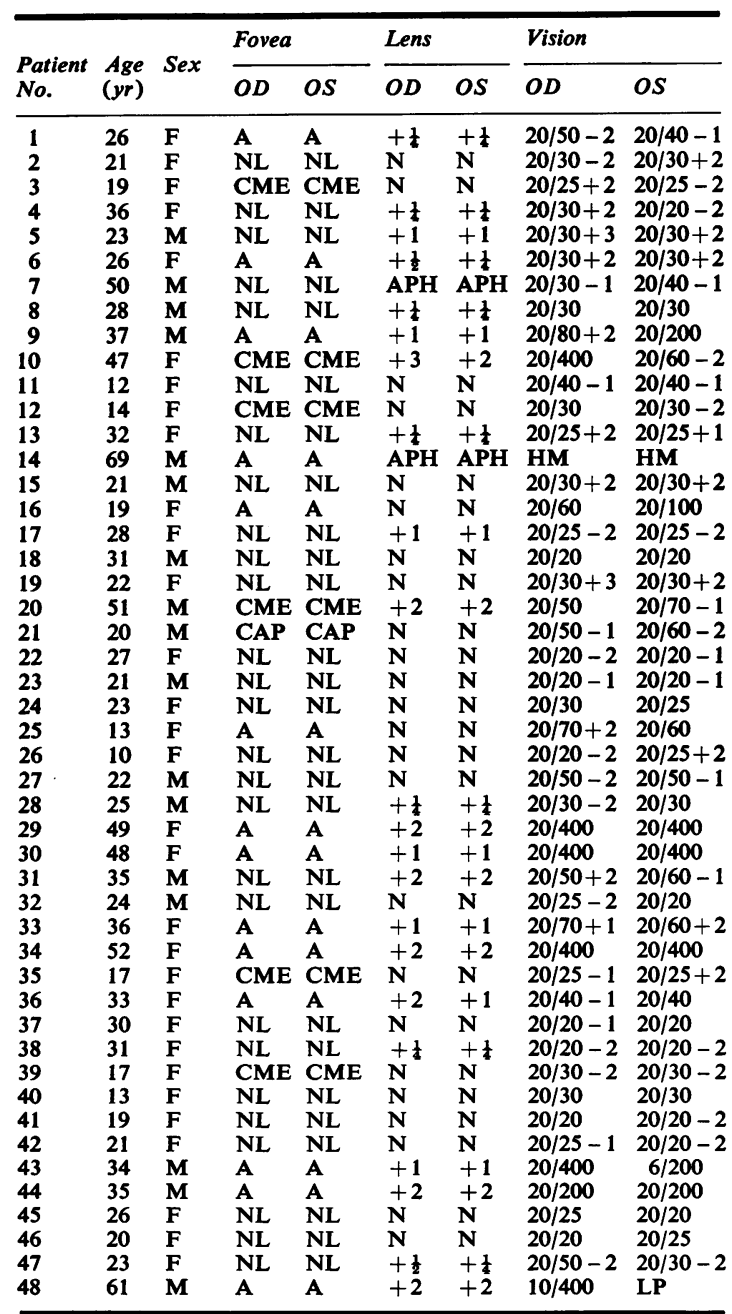

*A indicates atrophic-appearing lesion; NL, no foveal lesion; CME, cystoid macular oedema; CAP, cystic-appearing foveal lesion; $N$, no lens opacity; APH, aphakic; HM, hand motions; and LP, light perception

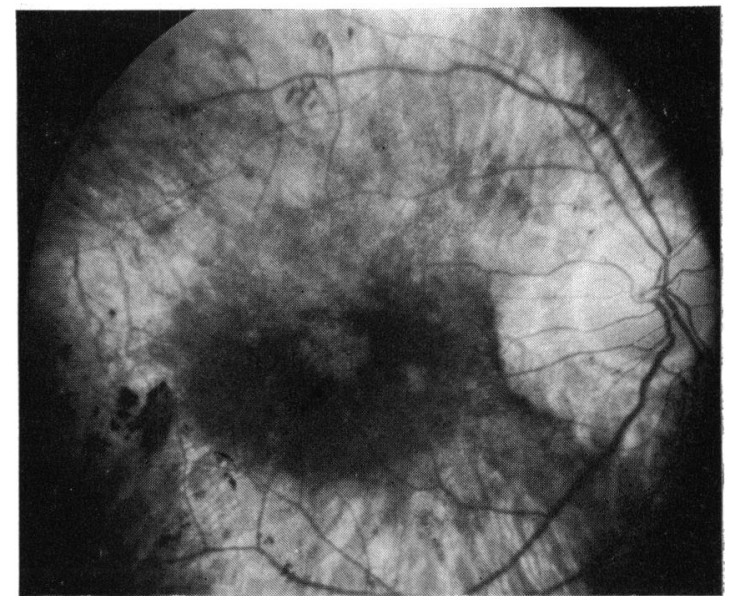

Fig. 2 Atrophic-appearing foveal lesion in the right eye of patient with Usher's syndrome

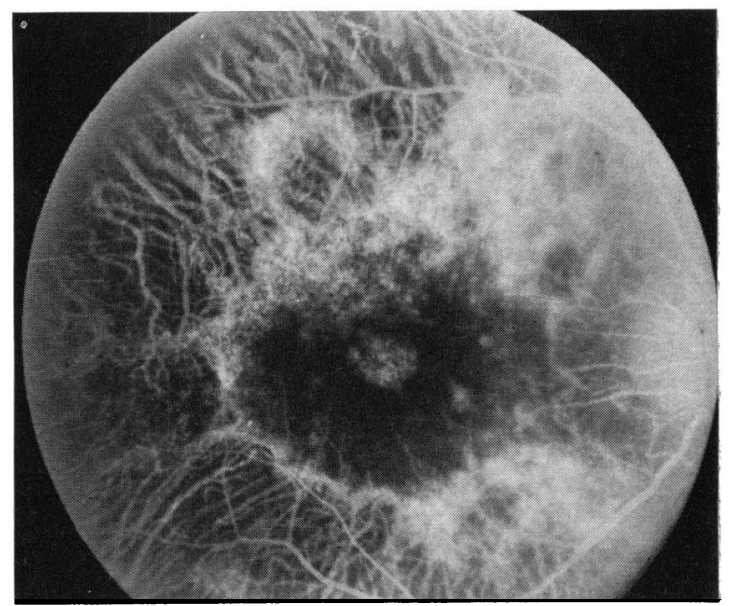

Fig. 3 Fluorescein angiogram from same patient shown in Fig. 2. Note foveal hyperfluorescence corresponding to atrophic foveal lesion. Extensive atrophy of choriocapillaris vessels outside fovea is also shown

mately $5 \mathrm{~mm}$ in diameter that projects perimetrically $16^{\circ}$ to $20^{\circ}$ of the central visual field.

Twenty-seven patients $(56 \%)$ showed no lesions within the fovea of either eye by ophthalmoscopy, colour photography, or fluorescein angiography. Fourteen patients $(29 \%)$ showed bilateral atrophicappearing foveal lesions, as noted in Figs. 2 and 3. The atrophic foveal changes in 5 of these 14 patients were subtle, being convincingly apparent only on slit-lamp contact lens examination and fluorescein angiography. An atrophic-appearing foveal change was, with two exceptions, accompanied by a significant degree of reduction in central acuity. Two patients (1 and 6) with subtle atrophic-appear- 


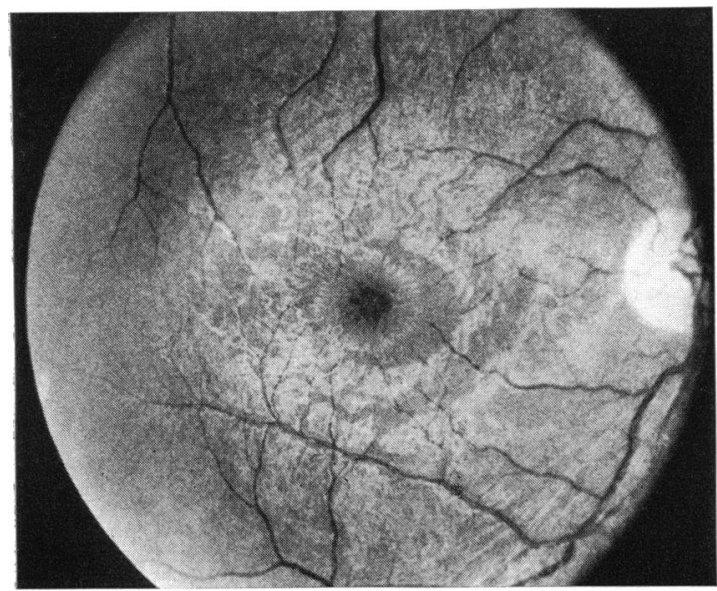

Fig. 4 Cystic-appearing foveal lesions in right eye. Note extensive shimmering appearance of perifoveal region associated with changes of internal limiting membrane

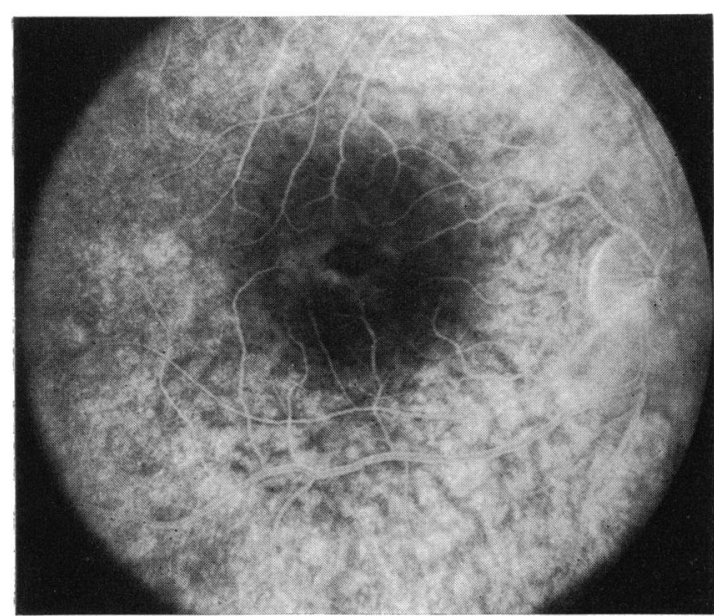

Fig. 6 Fluorescein angiogram from right eye of patient with cystoid macular oedema. Note leakage from perifoveal capillaries

ing foveal lesions showed central visual acuities between $20 / 30$ and 20/50 in each eye. These patients did not, however, show atrophic changes directly within the foveola but rather towards the margin of the fovea. The remaining 12 patients showed atrophic-appearing changes that involved the foveola, and they had visual acuities of $20 / 60$ or less in each eye.

One patient $(2 \%)$ showed bilateral cystic-appearing foveal lesions that were not associated with leakage of fluorescein dye from perifoveal capillaries on angiography (Figs. 4 and 5). Since an intact internal limiting membrane was not evident on slit-lamp

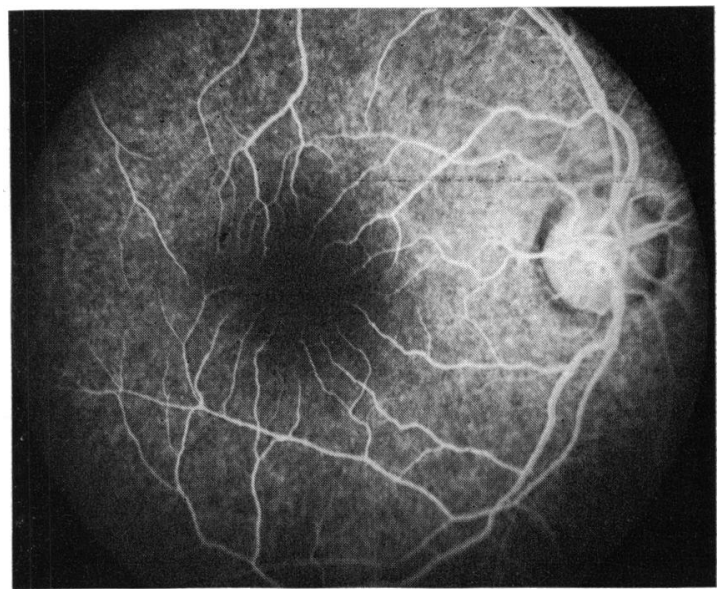

Fig. 5 Fluorescein angiogram from patient shown in Fig. 4. No fuorescein dye leaks from perifoveal retinal capillaries

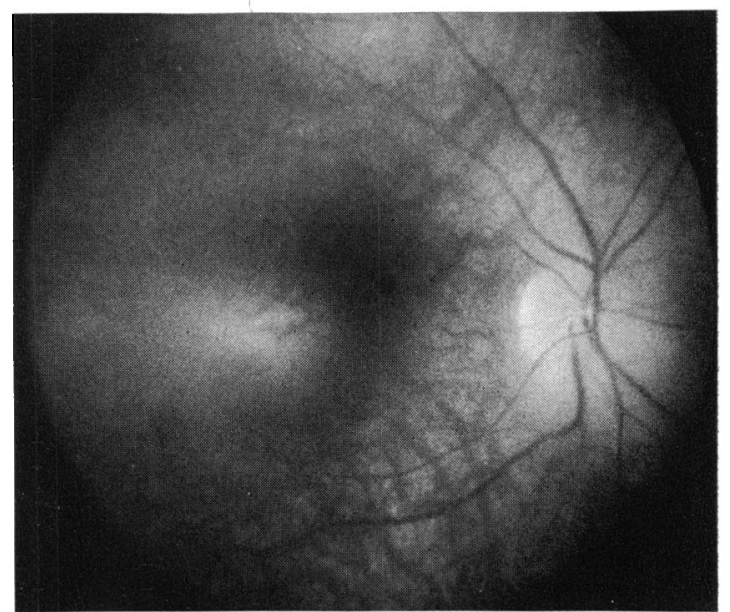

Fig. 7 Late stage angiogram from same patient shown in Fig. 6. Note accumulation of dye within cystoid spaces. Black spot in centre of photograph is artefact

biomicroscopy, these cystic-appearing lesions would most accurately be classified as partial-thickness holes. Even in the absence of lens changes visual acuity was reduced to $20 / 50$ and 20/60 in the right and left eyes, respectively. Six additional patients $(13 \%)$ showed cystic-appearing lesions accompanied by leakage from perifoveal retinal capillaries. In 4 of the 6 patients the cystic changes were subtle enough to be missed on superficial examination by direct ophthalmoscopy and were only suspected by contact lens examination and subsequently confirmed by fluorescein angiography (Figs. 6 and 7). In the absence of lens opacities in 4 of these 6 
Fig. 8 Scattergram showing degree of visual loss with age in 48 patients with Usher's syndrome. The numbers 2, 4, and 6 represent distance in inches. Symbols $\bar{c} p$ and $\bar{s} p$ indicate with and without correction, respectively. Horizontal line shows 20/200 level of central acuity

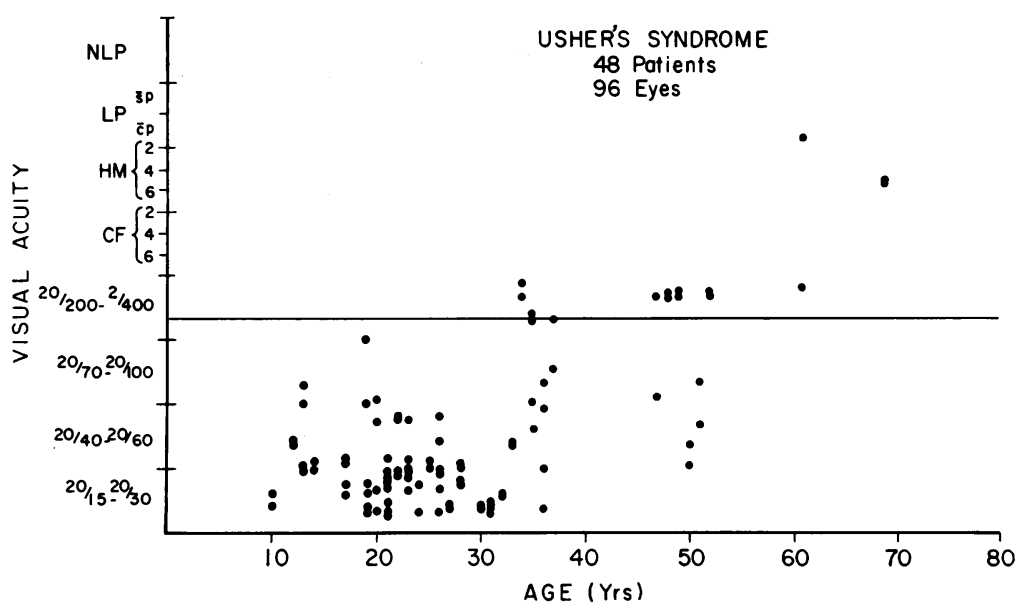

patients the presence of cystoid macular oedema did not compromise central visual acuity to levels below $20 / 30$.

The degree of visual loss with age is plotted in Fig. 8. Central visual acuity to $20 / 60$ or better is retained in the majority of patients until the mid-30s. Although enough representative cases are not available beyond the age of 40 years, there is a trend toward a significant reduction in central acuity to levels of $20 / 200$ or less. Note, however, that in 3 patients ( 5 eyes), central acuity of 20/70 or better was evident until the early 50 s. Until age 40 only 5 of 82 eyes showed visual loss of $20 / 200$ or less, while beyond that age 11 of 16 eyes showed a loss to levels of $20 / 200$ or less.

Lens changes are recorded in Table 1. Not unexpectedly there was a trend toward increasing degrees of lens opacities with age. The degree to which lens opacities compromised visual acuity in individual patients could not be assessed with any reliable degree of accuracy. Nevertheless, with appropriate cataract surgery, it is likely that the potential for visual performance, as depicted in Fig. 8, is somewhat better than actually represented because of the influence of posterior subcapsular lens opacities.

\section{Discussion}

An atrophic-appearing foveal lesion is the most frequently found fundus alteration associated with significant loss of central visual acuity in patients with Usher's syndrome. If the atrophic changes spare the foveola, less extensive loss in central acuity occurs. Additionally, a less frequent finding that is associated with at least a moderate reduction in central acuity is partial-thickness foveal holes. In our patients the presence of cystoid macular oedema did not compromise central acuity beyond the level of 20/30 when posterior subcapsular lens opacities were absent.

As a general finding patients with milder degrees of foveal pigment atrophy that spared the foveola tended to show either no or less-extensive $(+1$ or less; Table 1) posterior subcapsular lens opacities. When grossly apparent atrophic-appearing foveal lesions with foveolar involvement were found, the degree of lens changes were generally more extensive $(+2$ or greater; Table 1$)$. These latter patients were, as a group, older than those with less extensive atrophic-appearing foveal lesions and less-extensive lens opacities. Four of the 6 patients with cystoid macular oedema were less than 20 years old and showed no clinical evidence of posterior subcapsular lens opacities. The other 2 patients were 47 years and 51 years, respectively, and both showed extensive $(+2$ or greater) posterior subcapsular cataractous changes.

The data shown in Fig. 8, which depict visual loss with age, are helpful as a general guide when counselling patients with Usher's syndrome as to the potential for visual loss with age. This type of information has important implications for vocational training.

The value of such data would be enhanced by the pooling of similar observations from several different investigators if a larger sampling within older, as well as younger, age groups could be obtained.

This work was supported in part by an unrestricted grant from Research to Prevent Blindness Inc., NYC, and by a research grant from the Illinois Society for the Prevention of Blindness, Chicago.

\section{References}

Abraham, F. A., Cohen, D., and Sohmer, H. (1977). Usher's syndrome: electrophysiological tests of the visual and 
auditory systems. Documenta Ophthalmologica, 44, 435-444.

Bergsma, D. R. (1976). The Usher syndrome: clinical definition and related research. In Workshop on Usher's Syndrome, pp. 8-14. Edited by N. L. Tully. Helen Keller National Center: New York.

Fishman, G. A., Fishman, M., and Maggiano, J. (1977a). Macular lesions associated with retinitis pigmentosa. Archives of Ophthalmology, 95, 798-803.

Fishman, G. A., Maggiano, J., and Fishman, M. (1977b). Foveal lesions seen in retinitis pigmentosa. Archives of Ophthalmology, 95, 1993-1996.

Hallgren, B. (1959). Retinitis pigmentosa combined with congenital deafness; with vestibulo-cerebellar ataxia and mental abnormality in a proportion of cases: a clinical and genetics-statistical study. Acta Psychiatrica Scandinavica, Supplement, 138, 5-101.

Merin, S., Abraham, F. A., and Auerbach, E. (1974). Usher's and Hallgren's syndromes. Acta Geneticae Medicae et Gemellologiae, 23, 49-55.

Usher, C. H. (1914). On the inheritance of retinitis pigmentosa with notes of cases. Royal London Ophthalmologic Hospital Reports, 19, 130-236.

von Graefe, A. (1858). Exceptionelles Verhalten des Gesichtsfeldes bei pigmententartung der Netzhaut. Albrecht von Graefes Archiv für klinische und experimentelle Ophthalmologie, 4, 250-253. 